\title{
Evaluation of transvaginal sonography in detecting endometrial polyps and the pregnancy outcome following hysteroscopic polypectomy in infertile women
}

\author{
HUILI ZHU, JING FU, HAIKE LEI, YONG SONG, LICONG SHEN and WEI HUANG \\ Department of Gynecology and Obstetrics, West China Second University Hospital, \\ Sichuan University, Chengdu, Sichuan 610041, P.R. China
}

Received March 12, 2015; Accepted April 18, 2016

DOI: $10.3892 / \mathrm{etm} .2016 .3407$

\begin{abstract}
The aims of the present study were to evaluate the effectiveness of transvaginal sonography (TVS) in the detection of endometrial polyps (EPs), and to assess the pregnancy outcome in infertile women following hysteroscopic polypectomy. A total of 145 women diagnosed with primary or secondary infertility and intrauterine disorders by TVS and hysterosalpingography (HSG) were included in the current study. All subjects were divided into three groups based on hysteroscopic findings, including the EP, intrauterine adhesion and normal groups. EPs were removed for biopsy and intrauterine adhesions were treated. Pregnancy rates between groups were compared. In total, 34 EPs were detected by TVS, while 45 subjects were later confirmed with EP by hysteroscopy. The sensitivity, specificity, positive predictive value and negative predictive value of TVS in the detection of EPs were $67,96,88.23$ and $86.49 \%$, respectively. Of the included patients, 120 subjects were followed up, including 40 patients diagnosed with EPs, 42 with intrauterine adhesions and 38 with normal cavities. The results indicated no statistically significant differences in the age, type and duration of infertility, least function (LF) score and classification of the extent of tubal disease with the distal fimbrial obstruction between the three groups. In addition, pregnancy rate and spontaneous abortion rate in the EP group following hysteroscopic polypectomy were 45 and $5.6 \%$, respectively. No significant difference was observed in the fertility rate following surgery. In conclusion, TVS features high sensitivity, specificity and certain unique sonographic characteristics in diagnosing EPs, and may be used as a preliminary diagnostic procedure to select patients for hysteroscopy. Furthermore, hysteroscopic polypectomy is
\end{abstract}

Correspondence to: Dr Wei Huang, Department of Gynecology and Obstetrics, West China Second University Hospital, Sichuan University, 20 South Renmin Road, Chengdu, Sichuan 610041, P.R. China

E-mail: weihuangcn@126.com

Key words: transvaginal ultrasonography, endometrial polyp, hysteroscopic polypectomy, pregnancy rate an important approach for the treatment of infertile patients with EPs and appears to help increase the pregnancy rate of previously infertile women.

\section{Introduction}

Endometrial polyps (EPs) are common during the reproductive years, occurring in up to $24 \%$ of women, and their incidence increases with age (1). EPs are formed due to proliferation and hypertrophy of the basal layer of the endometrium, and the risk of malignancy is variable (2). A number of studies have investigated the association of infertility and EPs $(3,4)$; however, the interference of EPs in the fertility potential remains unknown. Certain studies have suggested that women with polyps have a higher rate of miscarriage, although there is no evidence of lower pregnancy rates in these women (5). It has been suggested that hysteroscopic polypectomy should be performed in infertile women (6); however, the importance of routine hysteroscopic removal of polyps remains unknown.

Techniques for the diagnosis of EP have improved in recent years; however, the real incidence of EP should be higher than it is known since numerous polyps are asymptomatic. EPs are often detected during the investigation of menstrual cycle disorders, or upon evaluation of an infertility case using transvaginal sonography (TVS) or hysterosalpingography (HSG) $(7,8)$. Hysteroscopy is a highly reliable and sensitive tool for the detection of intrauterine disorders (9). Upon hysteroscopy examination, EPs appear soft and smooth, and often present only a small degree of vascularization. However, due to the invasiveness and cost of hysteroscopy, it may be the initial technique offered to patients. While previous ultrasonographic studies have primarily investigated EPs in patients with abnormal uterine bleeding $(10,11)$, the association between intrauterine lesions and infertility remains unclear. The utility of TVS compared with other methods in diagnosing intrauterine disorders has drawn increased attention of researchers, however, its usefulness in detecting EPs in infertile women remains rarely discussed (12). Therefore, the aims of the present study were to evaluate the significance of TVS in detecting EP and to assess the pregnancy outcome following hysteroscopic polypectomy in infertile women. 


\section{Materials and methods}

Patients. Between March 2011 and December 2011, a total of 145 women diagnosed with primary or secondary infertility and intrauterine disorders by TVS and HSG at the West China Second Hospital (Sichuan University, Chengdu, China) were selected and included for study. All subjects met the following inclusion criteria: Age of $\leq 40$ years; and at least 12 months of infertility, without any other complications. All subjects were followed up by phone or correspondence for their reproductive histories. In total, 120 subjects were followed up by phone or correspondence for their reproductive histories, while 25 subjects were not able to be followed up due to incorrect or outdated contact information. The patients were followed up once a year for three years to investigate their pregnancy outcome. No significant difference was detected in the age or type of infertility between patients followed up and those not. The present study was conducted in accordance with the Declaration of Helsinki, and with approval from the Ethics Committee of Sichuan University. Written informed consent was obtained from all the participants.

Diagnostic technique and surgery. Prior to hysteroscopy, TVS and HSG were performed for the initial assessment of intrauterine abnormalities. Irregularities in the size and shape of uterus, the thickness of the endometrial stripe, endometrial cavity contours and echo patterns of endometrium in the transverse plane and the long axis were considered during TVS (13). Filling defects in the uterine cavity and irregularities in the uterine wall were considered during HSG (14). Subsequently, all 145 subjects were examined by laparoscopy (A22001A 4 mm Telescope; Olympus Winter \& IBE GmbH, Hamburg, Germany) in combination with hysteroscopy as part of their routine infertility evaluation. Depending on their laparoscopic and hysteroscopic results, patients underwent procedures to remove possible infertility factors in the first half of their menstrual cycle. All EPs identified by hysteroscopy were removed by hysteroscopic polypectomy and sent for independent histopathological examination for confirmation by the Department of Pathology. Transvaginal sonography examinations were performed with Aloka SSD-1000 ultrasound system (Hitachi Aloka Medical, Ltd., Tokyo, Japan) and real-time scanners with $5.0 \mathrm{MHz}$ endovaginal transducers (Hitachi Aloka Medical, Ltd.). For HSG, 5-10 ml water-soluble contrast medium [Pielograf 70\% (amidotrizoato meglumine); Juste S.A.Q.F., Madrid, Spain] was introduced into the uterine cavity after placing a balloon catheter set. The soft rubber Foley catheter was inflated in the cervical canal under fluoroscopic control using a digital system (MultiDiagnost 3; Philips Medical Systems Nederland B.V., Best, The Netherlands). Hysteroscopy was performed with an 8 -mm hysteroscope (Uteromat Fluid Control/A4060; Olympus Winter \& IBE $\mathrm{GmbH}$ ) with an 8.5-mm outer diameter that provides an oblique view of a 12 degree gradient with the optical axis. Distention of the uterine cavity during hysteroscopy was accomplished by normal saline insufflation (13). Classification of the extent of tubal disease (CETD) and least function (LF) score were employed during or at conclusion of surgery to assess each patient's tubal function.
Based on the hysteroscopy findings, the participants were divided into three groups, including the EP, intrauterine adhesion and normal groups. The cavity was systematically inspected for any abnormal findings. EP was localized in relation with the tubal ostia and the uterine walls or fundus. According to the size and number of EPs, patients in the EP group were further divided into two subgroups, including patients with an EP $<1 \mathrm{~cm}$, or patients with an EP $\geq 1 \mathrm{~cm}$ or with multiple EPs. The pregnancy rates were compared between these two EP subgroups.

Statistical analysis. Analysis of variance was employed to compare the age, infertility duration, LF score (15) and CETD (16). Survival analysis (Kaplan-Meier) was performed to ascertain the probability of conceiving in the three groups. Curves were compared by means of Mantel-Haenszel log-rank test for categorical variables. The effect of various covariates on fertility was estimated using Cox's proportional hazards model. $\chi^{2}$ test was employed to investigate the fertility rates of the three groups. Analyses were performed using the SPSS version 13.0 statistical package (SPSS, Inc., Chicago, IL, USA), with statistically significant differences indicated by $\mathrm{P}<0.05$.

\section{Results}

Diagnosis and follow-up. The ultrasound scans of EPs were mainly characterized by strong- or equal-echo mass in the uterine cavity, interruption in the continuity of the midline echo, or uneven echo displayed in the endometrium. The present study considered hysteroscopic results to be the gold standard for the diagnosis of EPs and intrauterine adhesions. In total, 34 subjects were found to present EP using TVS. The majority of patients were examined during the mid- and late follicular phase, and a few during the early follicular phase. The sensitivity, specificity, positive predictive value and negative predictive value of TVS in the detection of EPs were $67,96,88.23$ and $86.49 \%$, respectively. Compared with hysteroscopy as the gold standard, the sensitivity of TVS in detecting EP was relatively low; however, the two methods are similar in specificity, positive and negative prediction value. Though TVS may not replace hysteroscopy, it may be helpful to use TVS for initial screening of EP. When TVS does not indicate abnormalities, hysteroscopy may not be required.

In total, 120 of the 145 subjects were followed up, which included 40 patients diagnosed with EPs, 42 with intrauterine adhesions and 38 with normal cavities. The ages of patients in EP, normal and adhesions groups were $30 \pm 3.5,30.5 \pm 3.9$ and $31.3 \pm 3.3$ years, respectively. The LF scores of the three groups were $5.6 \pm 2.4,4.9 \pm 2.7$ and $4.9 \pm 2.0$, respectively. The CETD classes of the three groups were $1.9 \pm 1.4,2.0 \pm 0.9$ and $1.9 \pm 0.9$, respectively. The infertility duration of the three groups were $4.4 \pm 3.5,4.7 \pm 3.1$ and $4.4 \pm 2.7$ years, respectively. No significant differences in age, type and duration of infertility, LF and CETD were identified between the three groups, as shown in Table I.

The fertility rates of normal, EP and adhesion groups were $47.4,42.5$ and $33.3 \%$, respectively. No significant difference in fertility rate was observed between the three groups, as shown in Table I 
Table I. Factors associated with fertility.

\begin{tabular}{lcrcc}
\hline Factor & $\begin{array}{c}\text { EP } \\
(\mathrm{n}=40)\end{array}$ & $\begin{array}{c}\text { Normal } \\
(\mathrm{n}=38)\end{array}$ & $\begin{array}{c}\text { Adhesion } \\
(\mathrm{n}=42)\end{array}$ & P-value \\
\hline Age (years) & $30 \pm 3.5$ & $30.5 \pm 3.9$ & $31.3 \pm 3.3$ & 0.1 \\
LF score & $5.6 \pm 2.4$ & $4.9 \pm 2.7$ & $4.9 \pm 2.0$ & 0.06 \\
CETD & $1.9 \pm 1.4$ & $2.0 \pm 0.9$ & $1.9 \pm 0.9$ & 0.23 \\
ID (years) & $4.4 \pm 3.5$ & $4.7 \pm 3.1$ & $4.4 \pm 2.7$ & 0.14 \\
\hline
\end{tabular}

EP, endometrial polyp; LF, least function; CETD, classification of the extent of tubal disease; ID, infertility duration.

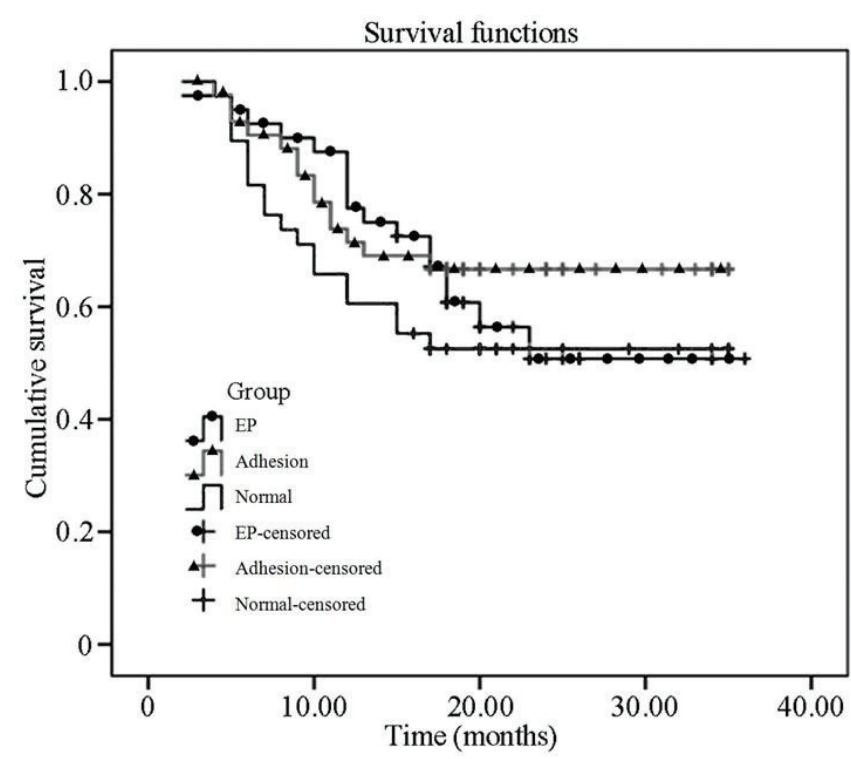

Figure 1. Kaplan-Meier survival analysis and Mantel-Haenszel log rank test. No statistically significant difference was observed in fertility rates between the three groups.

Survival analysis. Survival analysis showed that no statistically significant difference in fertility rate following surgery was observed between the EP, intrauterine adhesion and normal groups ( $\mathrm{P}>0.05$; Fig. 1) (Table II). Furthermore, no statistically significant difference was observed in accumulated pregnancy rates between the three groups $(P>0.05$; Fig. 2).

Factors affecting fertility. The effects of various covariates on fertility were estimated using Cox's proportional hazards model analysis. The results indicated that the age, infertility duration and CETD class did not appear to have any statistically significant influence on the pregnancy rate. However, the LF score was found to be negatively correlated with pregnancy rate (i.e., the higher LF score, the lower pregnancy rate).

Pregnancy rate and spontaneous abortion rate in the EP group following hysteroscopic polypectomy were identified to be 45 and 5.6\%, respectively. However, there was no statistically significant difference in the fertility rate between patients with polyps $<1 \mathrm{~cm}$ in size and patients with polyps $\geq 1 \mathrm{~cm}$ in or multiple polyps $\left(\chi^{2}=0.02 ; \mathrm{P}=0.96\right)$ (Table III).
Table II. Fertility rate in the three groups.

\begin{tabular}{lccc}
\hline Group & Fertility rate (\%) & $\chi^{2}$-test & P-value \\
\hline Normal $(\mathrm{n}=38)$ & 47.4 & & \\
EP $(\mathrm{n}=40)$ & 42.5 & $0.658^{\mathrm{a}}, 0.372^{\mathrm{b}}$ & $0.08^{\mathrm{a}}, 0.12^{\mathrm{b}}$ \\
Adhesion $(\mathrm{n}=42)$ & 33.3 & $1.772^{\mathrm{a}}$ & $0.01^{\mathrm{a}}$ \\
\hline
\end{tabular}

${ }^{a}$ Compared with the normal group; ${ }^{b}$ compared with the adhesion group. EP, endometrial polyp.

Table III. Fertility rates of patients with EPs.

\begin{tabular}{lccc}
\hline Group & Fertility rate $(\%)$ & $\chi^{2}$ test & P-value \\
\hline$<1 \mathrm{~cm}(\mathrm{n}=23)$ & 46.7 & & \\
$\geq 1 \mathrm{~cm}(\mathrm{n}=17)$ & 43.5 & 0.02 & 0.96 \\
\hline
\end{tabular}

Fertility rates of patients with EPs $<1 \mathrm{~cm}$ in size, $>1 \mathrm{~cm}$ in size or with multiple polyps. EP, endometrial polyp.

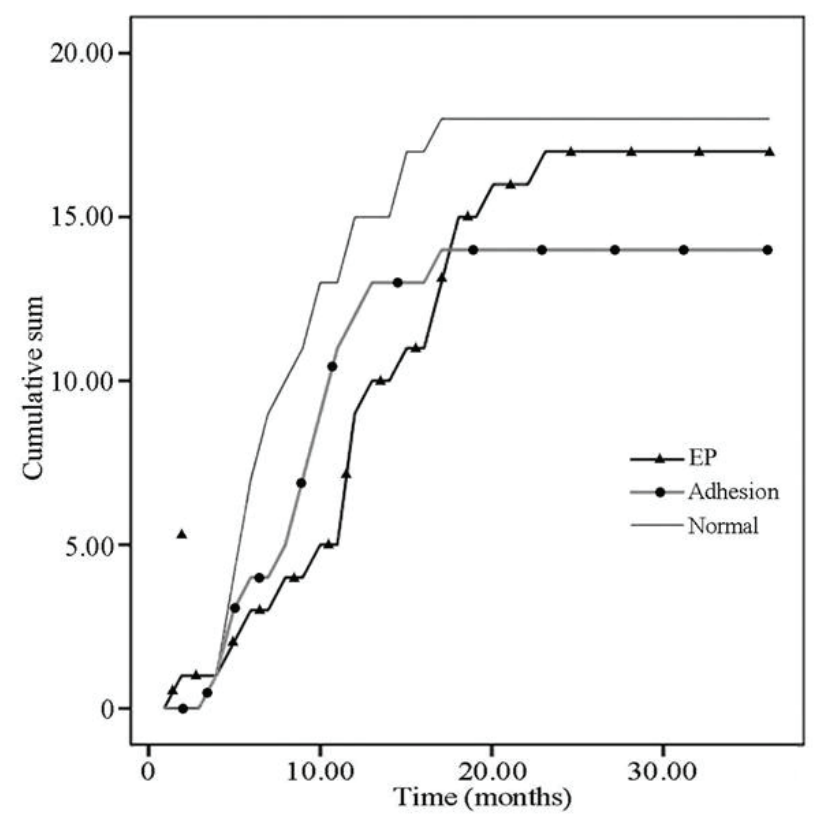

Figure 2. Accumulated pregnancy rates in the EP, intrauterine adhesion and normal groups. EP, endometrial polyp.

\section{Discussion}

EPs are frequently unrecognized, particularly in cases where they are not sufficiently large or are asymptomatic. TVS is a non-invasive modality that provides excellent imaging of the uterus and endometrial abnormalities $(17,18)$. This method is relatively painless and cheap, and is well accepted by patients. The appropriate time to perform a TVS examination of the endometrium is during the follicular phase. As the normal endometrium is thin, EP can be certainly recognized. In the present study, the sensitivity, specificity, positive predictive value and negative predictive value of TVS in the detection of 
EPs were 67, 96, 88.23 and $86.49 \%$, respectively. This result is similar to the findings of another study, in which the results of these parameters were 71.4, 100, 100 and 97.1\%, respectively (19). A study by Yantapant (20) examined 60 EP patients with a mean age of 31-40 years. In that study, sensitivity, specificity and accuracy of the diagnosis of EPs by TVS were 60, 33.3 and $57.6 \%$, respectively (20). Vitner et al (21) identified that, although hysteroscopy presented improved predictive values for diagnosing uterine polyps when compared with TVS, the difference was not statistically significant, and TVS had a significantly higher sensitivity in diagnosing retained products of conception. Though all these studies pointed to similar advantages of TVS, it should be noted that TVS may provide insufficient information in certain cases. For instance, small endometrial structures protruding into the cavity may be missed (22). The results also seem to be associated with the experience and skill of the physicians. Nevertheless, patients with normal findings on TVS do not require further examination by hysteroscopy, which will help reduce the high cost of hysteroscopy and morbidity. The morbidity rate of EP in infertile women is associated with the examination method employed and the cases studied. Ahmadi et al (23) previously reported a sensitivity of $91.4 \%$ and a specificity of $80.2 \%$ for three-dimensional hysterosonography in diagnosing polyps, respectively.

The high morbidity rate of EP in infertile women indicates that EP may interfere in the gestation. De Placido et al (24) considered 950 female candidates for an in vitro fertilization (IVF) program. Of these females, 602 cases were examined with a mini-hysteroscope and 348 women with a $5 \mathrm{~mm}$ hysteroscope, and EP was detected in $146(24.2 \%)$ and 82 cases $(23.6 \%)$, respectively (20). Similarly, a study by Silló-Seidl (25) reported the presence of polyps in $10.8 \%$ of patients in a series of 1,000 sterile patients, and pregnancy was achieved in 8 of these patients following polypectomy. By contrast, Hereter et al (26) investigated 33 patients with EP and 280 patients without EP, and identified no significant differences between the two groups with respect to implantation and abortion in IVF cycles. In addition, a study by Varasteh et al (27) in 23 sterile women reported a correlation between the polypectomy and the accumulated pregnancy rate of $65.2 \%$, while polypectomy in infertile women was likely to increase the pregnancy rate by a factor of 3-4 times. However, polyps and myomas were mixed in the study, and thus the conclusions drawn by the authors were questionable (27). In another series, 19 out of 25 infertile patients (76\%) undergoing polypectomy were able to conceive within a 12-month period (28). Furthermore, Stamatellos et al (29) reported that the total pregnancy rate subsequent to hysteroscopic polypectomy was $61.4 \%$, a percentage that is comparable with the aforementioned studies. Another study investigated intrauterine insemination (IUI) in infertile women whose only known problem was EP, and the proportion of patients achieving pregnancy in the EP group was $64 \%$, which was nearly twice that of the control group (30). In addition, the authors reported that pregnancies following polypectomy were frequently obtained spontaneously, while waiting for IUI. In the present study, the total pregnancy rate was $42.5 \%$, and the risk of spontaneous abortion was $5.6 \%$, which is less than the values previously reported (31), but is similar the value reported for the normal group. All the studies suggest that hysteroscopic polypectomy is an effective measure.

Certain studies identified a significant association of pregnancy rate with the size of myomas, as the probability of pregnancy increased proportionally with the size of the resected myoma (27,31). However, it has been suggested that asymptomatic polyps ( $<2 \mathrm{~cm}$ in diameter) do not appear to interfere with IVF and embryo transfer conception rates, but may increase the risk of spontaneous abortion (5). Persistent functional EPs are likely to impair fertility, even when these are small. Therefore, removal of such lesions may improve subsequent reproductive performance (32). Another study suggested that the hysteroscopic removal of small polyps improved the reproductive outcome, and it is recommended for infertile women to undergo assisted reproductive technology procedures $(30,33)$. These findings are similar with the results of the current study, since no association was observed between the polyp size and the chance of pregnancy. In addition, it is suggested that EPs contribute to infertility and therefore should be removed irrespective of their size or number. EP's interference with fertility does not appear to be associated with a space-occupying lesion mechanism. However, further studies are required to address this question.

In conclusion, TVS features high sensitivity, specificity and certain unique sonographic characteristics in diagnosing EPs. Due to the invasiveness and cost of hysteroscopy, TVS may be of interest for use as a preliminary diagnostic procedure to screen patients for hysteroscopy. Hysteroscopic polypectomy is an effective procedure for removing EPs. The mechanism by which EPs interfere with fertility potential remains unclear, hysteroscopic removal of EPs of any size appears to help improve the pregnancy outcome of infertile women

\section{Acknowledgements}

The authors would like to thank all the women who participated in the present study, as well as all the staff involved at the West China Second University Hospital.

\section{References}

1. Valle RF: Therapeutic hysteroscopy in infertility. Int J Fertil 29: 143-148, 1984.

2. Bakour SH, Khan KS and Gupta JK: The risk of premalignant and malignant pathology in endometrial polyps. Acta Obstet Gynecol Scand 81: 182-183, 2002.

3. Tanos V: Endometrial polyps and infertility. In: Reproductive Surgery in Assisted Conception. Metwally M and Li TC (eds). Springer London, London, pp219-221, 2015.

4. Bocca SM: Endometrial polyps. In: Ultrasound Imaging in Reproductive Medicine. Stadmauer LA and Tur-Kaspa I, Springer London, London, pp133-149, 2014.

5. Lass A, Williams G, Abusheikha $\mathrm{N}$ and Brinsden P: The effect of endometrial polyps on outcomes of in vitro fertilization (IVF) cycles. J Assist Reprod Genet 16: 410-415, 1999.

6. Shen L, Wang Q, Huang W, Wang Q, Yuan Q, Huang Y and Lei H: High prevalence of endometrial polyps in endometriosis-associated infertility. Fertil Steril 95: 2722-2724, 2011.

7. Haider Z, Alkatib M, Syed A and Bourne T: Transvaginal scan (TVS) versus TVS and saline infusion hydrosonography for the diagnosis of endometrial polyps and submucous fibroids. BJOG 113: 868-868, 2006.

8. Gao YY, Xin YL, Wei XQ, Hou QN and Zhang CL: A study on the diagnostic value of three examination methods for endometrial polyps in patients with infertility. Xian Dai Sheng Wu Yi Xue Jin Zhan 15: 3053-3057, 2015 (In Chinese). 
9. Farquhar C, Ekeroma A, Furness S and Arrol B: A systematic review of transvaginal ultrasonography, sonohysterography and hysteroscopy for the investigation of abnormal uterine bleeding in premnopausal women. Acta Obstet Gynecol Scand 82: 493-504, 2003

10. Timmermans A, Gerritse MB, Opmeer BC, Jansen FW, Mol BW and Veersema S: Diagnostic accuracy of endometrial thickness to exclude polyps in women with postmenopausal bleeding. J Clin Ultrasound 36: 286-290, 2008.

11. Dueholm M, Jensen ML, Laursen H and Kracht P: Can the endometrial thickness as measured by trans-vaginal sonography be used to exclude polyps or hyperplasia in pre-menopausal patients with abnormal uterine bleeding? Acta Obstet Gynecol Scand 80: 645-651, 2001.

12. Song Y, Shen LC, Huang W, Lei HK, Wang QS and Zhu HL: Diagnostic value of endometrial thickness determined by transvaginal sonography in infertile women with endometrial polyps. Chin Med J (Engl) 125: 2279-2283, 2012.

13. Balić D and Balić: Office hysteroscopy, transvaginal ultrasound and endometrial histology: A comparison in infertile patients. Acta Medica Academica 40: 34-38, 2011.

14. Roma Dalfó A, Ubeda B, Ubeda A, Monzón M, Rotger R, Ramos R and Placio A: Diagnostic value of hysterosalpingography in the detection of intrauterine abnormalities: A comparison with hysteroscopy. American Journal of Roentgenology 183: 1405-1409, 2004.

15. Adamson GD and Pasta DJ: Pregnancy rates can be predicted by validated endometriosis fertility index (EFI). Fertil Steril 77 (Suppl 1): S48, 2002.

16. Rock JA, Katayama KP, Martin EJ, Woodruff JD and Jones HW Jr: Factors influencing the success of salpingostomy techniques for distal fimbrial obstruction. Obstet Gynecol 52: 591-596, 1978.

17. de Jong P, Doel F and Falconer A: Outpatient diagnostic hysteroscopy. Br J Obstet Gynaecol 97: 299-303, 1990.

18. Granberg S, Wickland M, Karlsson B, Norström A and Friberg LG: Endometrial thickness as measured by endovaginal ultrasonography for indetifying endometrial abnormality. Am J Obstet Gynecol 164: 47-52, 1991.

19. Shalev J, Meizner I, Bar-Hava I, Dicker D, Mashiach R and Ben-Rafael Z: Predictive value of transvaginal value of transvaginal sonography performed before routing diagnostic hyseroscopy for evaluation of infertility. Fertil Steril 73: 412-417, 2000

20. Yantapant A: Comparison of the accuracy of transvaginal sonography and hysteroscopy for the diagnosis of endometria polyps at Rajavithi Hospital. J Med Assoc Thai 95 (Suppl 3): S92-S97, 2012.
21. Vitner D, Filmer S, Goldstein I, Khatib N and Weiner Z: A comparison between ultrasonography and hysteroscopy in the diagnosis of uterine pathology. Eur J Obstet Gynecol Reprod Biol 171: 143-145, 2013.

22. Mendelson EB, Bohm-Velez M, Josef N and Neiman HL: Endometrial abnormalities: Evaluation with transvaginal sonography. AJR Am J Roentgenol 150: 139-142, 1988.

23. Ahmadi F, Rashidy Z, Haghighi H, Akhoond M, Niknejadi M, Hemat $M$ and Shamsipour M: Uterine cavity assessment in infertile women: Sensitivity and specificity of three-dimensional hysterosonography versus hysteroscopy. Iran J Reprod Med 11: 977-982, 2013.

24. De Placido G, Clarizia R, Cadente C, Castaldo G, Romano C, Mollo A, Alviggi C and Conforti S: Compliance and diagnostic efficacy of mini-hysteroscopy versus traditional hysteroscopy in infertility investigation. Eu J Obstet Gynecol Reprod Biol 135: 83-87, 2007.

25. Silló-Seidl G: The analysis of the endometrium of 1,000 sterile women. Hormones 2: 70-75, 1971.

26. Hereter L, Carreras O, Pascual MA, Martinez F and Barri PN: Repercussion of the presence of endometrial polyps in an I.V.F. cycle. Minerva Medica 29: 1071-1074, 1998.

27. Varasteh NN, Neuwirth RS, Levin B and Keltz MD: Pregnancy rates after hysteroscopic polypectomy and myomectomy in infertile women. Obstet Gynecol 94: 168-171, 1999.

28. Spiewankiewicz B, Stelmachów J, Sawicki W, Cendrowski K, Wypych P and Swiderska K: The effectiveness of hysteroscopic polypectomy in cases of female infertility. Clin Exp Obstet Gynecol 30: 23-25, 2003

29. Stamatellos I, Apostolides A, Stamatopoulos P and Bontis J: Pregnancy rates after hysteroscopic polypectomy depending on the size or number of the polyps. Arch Gynecol Obstet 277: 395-399, 2008.

30. Pérez-Medina T, Bajo-Arenas J, Salazar F, Redondo T, Sanfrutos L, Alvarez P and Engels V: Endometrial polyps and their implication in the pregnancy rates of patients undergoing intrauterine insemination: A prospective, randomized study. Hum Reprod 20: 1632-1635, 2005.

31. Fernandez H, Sefrioui O, Virelizier C, Gervaise A, Gomel V and Frydman R: Hysteroscopic resection of submucosal myomas in patients with infertility. Hum Reprod 16: 1489-1492, 2001.

32. Shoekir TA, Shalan HM and El Shafei MM: Significance of endometrial polyps detected hysteroscopically in eumenorrhoeic infertile women. J Obstet Gynaecol Res 30: 84-89, 2004.

33. Preutthipan S and Herabutya Y: Hysteroscopic polypectomy in 240 premenopausal and postmenopausal women. Fertil Steril 83 705-709, 2005. 